\title{
Modulation of Polyamine Biosynthesis in Transformed Tobacco Plants by Targeting Ornithine Decarboxylase to an Atypical Subcellular Compartment
}

\author{
G. Nölke ${ }^{1}$, B. Schneider ${ }^{1, \#}$, S. Agdour ${ }^{1}$, J. Drossard ${ }^{2}$, R. Fischer ${ }^{1,2}$, S. Schillberg ${ }^{2, *}$ \\ ${ }^{1}$ Institut für Molekulare Biotechnologie, RWTH Aachen, Germany; ${ }^{2}$ Fraunhofer Institute for Molecular Biology and \\ Applied Ecology IME, Germany and ${ }^{\#}$ Present address: Biologische Bundesanstalt, Institut für Pflanzenschutz im \\ Obstbau, Germany
}

\begin{abstract}
Ornithine decarboxylase (ODC) is a cytosolic enzyme that catalyses the direct decarboxylation of L-ornithine to putrescine, one of the rate-limiting steps of polyamine biosynthesis in plants. We targeted recombinant human ODC to the cytosol and apoplast of transformed tobacco (Nicotiana tabacum) plants, and evaluated the impact of subcellular compartmentalization on the accumulation of the enzyme and its corresponding metabolic product. Immunoblot analysis showed that human ODC accumulated to high levels in both the cytosol and apoplast of transiently transformed tobacco leaves. In stably transformed tobacco plants with ODC targeted to the apoplast, enzyme activity increased by up to $32-$ fold $(\mathrm{P}<0.001)$ and putrescine levels increased by up to 8.5 -fold $(\mathrm{P}<0.05)$ compared to wild type plants. These results demonstrate that the subcellular targeting of polyamine pathway enzymes may provide a useful strategy to enhance the accumulation and activity of enzymes involved in polyamine biosynthesis and may increase metabolic flux toward desired end products.
\end{abstract}

Key Words: metabolic engineering, polyamine metabolism, recombinant enzyme, subcellular targeting, transgenic plants.

\section{INTRODUCTION}

Polyamines are ubiquitous, low-molecular-weight, polycationic compounds involved in crucial developmental, physiological and metabolic processes [1]. The polyamine biosynthesis pathway is complex and regulated at multiple levels [2]. In plants, the polyamine putrescine can be synthesized from either ornithine or arginine through the activities of ornithine decarboxylase (ODC) and arginine decarboxylase (ADC), respectively. In a step common to most organisms, spermidine is formed from putrescine by the addition of an aminopropyl group donated by decarboxylated Sadenosylmethionine. This reaction is catalysed by spermidine synthase (SPDS), an aminopropyl-transferase. Spermine is formed by the addition of a second aminopropyl moiety to spermidine, catalyzed by another aminopropyl transferase, spermine synthase (SPMS) [3].

Previous analysis of the polyamine biosynthetic pathway using over-expression and antisense suppression techniques [4] have shown that polyamine synthesis in plants is under strict homeostatic regulation with regard to end-product accumulation [5-7]. In most cases, the overexpression of ADC or ODC has only a limited impact on the levels of spermidine and spermine, despite elevated levels of putrescine in transgenic plants. Therefore, it has been suggested that the polyamine biosynthesis pathway in plants is so rigidly controlled at multiple levels that an increased flux towards a

\footnotetext{
*Address correspondence to this author at the Fraunhofer Institute for Molecular Biology and Applied Ecology IME, Germany;

E-mail: stefan.schillberg@ime.fraunhofer.de
}

desired end product cannot be achieved by the modulation of single key enzymes $[6,8,9]$.

The objective of this study was to investigate how the subcellular localisation of recombinant ODC influences recombinant protein accumulation, enzyme activity and polyamine levels. Human odc was selected because the cDNA is well characterized, and the use of mammalian sequences avoids the cosuppression of endogenous genes in transgenic plants. Moreover, previous studies have indicated that overexpression of a mammalian derived odc gene resulted in increased ODC activity and putrescine production in plants [10-13].

Therefore, targeting enzymes to an atypical subcellular compartment provides a novel strategy for creating additional capacities for rate-limiting steps of a pathway. The feasibility of such a strategy has been illustrated for the biosynthesis of amino acids $[14,15]$ and terpenoid indole alkaloids in plants [16]. In the presented study, we demonstrated that targeting of recombinant ODC to a different and atypical subcellular compartment resulted in significant increased enzyme activity and putrescine levels. The transfer of biosynthesis steps to another subcellular compartment may provide an alternative mechanism for polyamine pathway manipulation, and may further increase our understanding of the regulation of polyamine biosynthesis in plants.

\section{MATERIAL AND METHODOLOGY}

\section{Plasmid DNA, Bacteria and Plants}

We used the following plasmid DNA, bacteria and plants: Vectors pGEM (Pharmacia, Freiburg, Germany) and 
pSS [17]; Escherichia coli strains DH5 $\alpha$ and SCS110 (Stratagene, Heidelberg) and Agrobacterium tumefaciens GV3101 strain (pMP90RK, Gm ${ }^{\mathrm{R}}, \mathrm{Km}^{\mathrm{R}}$, and $\mathrm{Rif}^{\mathrm{R}}$ ) [18]; tobacco (Nicotiana tabacum cv Petite Havana SR1). Plants were cultivated in a greenhouse in DE73 standard soil with a 16-h natural daylight photoperiod, $25 / 22^{\circ} \mathrm{C}$ day/night temperature. Leaves from 3-6-week old plants were used for vacuum infiltration.

\section{Construction of the Plant Expression Cassettes}

The gene encoding ODC was amplified from a human prostate cDNA library (Invitrogen, Karlsruhe, Germany) using primers based on the 5' and 3' sequences of the human odc (GenBank accession number M16650). Restriction sites EcoRI/NcoI and SalI/HindIII were introduced into the forward (5' CCG GAA TTC CCC ATG GGT AAC AAC TTT GGT AAT GAA GAG T 3') and reverse primer (5' GGG AAG CTT GTC GAC CAC ATT AAT ACT AGC CGA AGC A 3'), respectively. The sequence of $o d c$ was verified by sequence analysis.

The amplified PCR product was initially cloned as NcoI/SalI fragment into pGEM derivatives containing the 5' untranslated region of the chalcone synthase gene, an $\mathrm{N}$ terminal signal sequence for apoplastic targeting and a Cterminal c-myc or His6 tag (ODC-apo) [19]. Subsequently, the recombinant odc cDNA, including the $5^{\prime}$ targeting sequences and the 3' tags, was subcloned as EcoRI/SalI or EcoRI/XbaI fragments into the pSS plant expression vector [17] between the constitutive double enhanced CaMV 35S promoter and the CaMV terminator sequence, resulting in plant expression vectors pODC-cyt for cytosolic targeting as well as pODC-apo for secretion to the plant cell apoplast (Fig. 1).

\section{Transformation of Tobacco Plants}

The plant expression vectors were introduced into $A$. $t u$ mefaciens GV3101 cells by electroporation using a Gene Pulser II system (BioRad, Hercules, CA) according to the manufacturer's instructions. Recombinant ODC accumulation was evaluated using a transient expression assay of vacuum infiltrated tobacco leaves with recombinant agrobacteria $[19,20]$. Briefly, young leaves (approximately 6-12 cm in length) were selected from different plants, infiltrated with recombinant agrobacteria and incubated in sealed trays on wet paper (Whatman, Clifton) at $25^{\circ} \mathrm{C}$ with a 16 -h photoperiod for 24-72 h. After incubation, the leaves were weighted, frozen in liquid nitrogen, and stored at $-80^{\circ} \mathrm{C}$ until further processing.

Stably transformed N. tabacum cv. Petit Havana SR1 plants were generated by leaf disc transformation with recombinant $A$. tumefaciens. Transgenic $\mathrm{T}_{0}$ plants were regenerated from transformed callus [21]. For selection of kanamycin-resistant $T_{1}$ or $T_{2}$ plants, seeds or $T_{1}$ plants were collected and germinated on MSMO agar medium (Sigma, Deisenhofen, Germany) supplemented with $2 \%(\mathrm{w} / \mathrm{v}) \mathrm{su}-$ crose, $0.4 \mu \mathrm{g} / \mathrm{ml}$ thiamine, $0.4 \mu \mathrm{g} / \mathrm{ml}$ glycine, $0.1 \mu \mathrm{g} / \mathrm{ml}$ nicotinic acid, $0.1 \mu \mathrm{g} / \mathrm{ml}$ pyridoxine and $75 \mu \mathrm{g} / \mathrm{ml}$ kanamycin. To facilitate the selection of homozygous transgenic lines, seeds from selfed ODC producing $\mathrm{T}_{0}$ plants were plated on kanamycin-containing medium to test for segregation of the T-DNA loci. Plants from $\mathrm{T}_{1}$ lines showing 3:1 segregation ratios of kanamycin resistance to sensitivity were selfed to generate $T_{2}$ lines uniformly resistant to kanamycin. The non segregating seed was assumed to be homozygous and was used for further biochemical characterization.

\section{Protein Extraction and Analysis}

Total soluble protein (TSP) was extracted from leaves transiently or stably expressing recombinant ODC using $2 \mathrm{ml}$ of plant extraction buffer $(200 \mathrm{mM}$ Tris- $\mathrm{HCl} \mathrm{pH} 7.5,5 \mathrm{mM}$ EDTA, $0.1 \mathrm{mM}$ DTT, $0.1 \%(\mathrm{v} / \mathrm{v})$ Tween 20) per gram fresh weight. Cell debris was removed by two rounds of centrifugation $\left(16,000 \mathrm{~g} / 4^{\circ} \mathrm{C} / 30 \mathrm{~min}\right)$ and the supernatant was subjected to SDS-PAGE followed by electroblotting onto nitrocellulose membranes (Hybond-C; Amersham Life Science, Braunschweig, Germany) and immunoblot analysis. The primary 9E10 anti-c-myc antibody (clone no. CRL-1729; American Type Culture Collection, Manassas, VA) was used to detect recombinant ODC targeted to the cytosol and apoplast. Goat anti-mouse IgG Fc-specific alkaline phosphatase-conjugated antibodies (Jackson ImmunoResearch, West Grove, PA) were used as secondary antibody followed by colour development using a solution of nitroblue tetrazolium/5-bromo-4-chloro-3-indolyl-phosphate (Pierce, Rockford, IL). Total soluble protein content of the leaf crude extract was determined in triplicate by the Bradford protein assay (Bio-Rad, München, Germany) using bovine serum albumin as standard. The accumulation levels of the plant produced ODC were analysed quantitatively by immunoblot against standard concentrations of bacterially expressed recombinant proteins.

\section{RNA Isolation and Northern Blot Analysis}

Total RNA was extracted from tobacco leaves as described [22]. Ten $\mu \mathrm{g}$ of total RNA was mixed 1:3 (v/v) with RNA loading buffer, denatured for $10 \mathrm{~min}$ at $65^{\circ} \mathrm{C}$ and separated in $1 \%(\mathrm{w} / \mathrm{v})$ agarose gel with $2.4 \mathrm{M}$ formaldehyde in MOPS buffer. The gel was run at 65 volts for $2 \mathrm{~h}$, then equilibrated twice in 20x SSC buffer for $15 \mathrm{~min}$. The transfer of RNA-samples from the agarose gel onto Hybond $\mathrm{N}^{+}$nylon membranes was performed by a downward alkaline capillary transfer for $3 \mathrm{~h}$ at room temperature [23]. Cross-linking of RNA samples to the membrane was achieved by UV irradiation. The membrane was prehybridized at $54^{\circ} \mathrm{C}$ for $2 \mathrm{~h}$ and hybridized overnight at the same temperature in standard hybridization solution containing $10 \mathrm{ng}$ of the ODC-DIGdUTP labelled probe. Detection of dioxigenin-labelled ODC was performed according to Bronstein [24].

\section{Preparation of Intercellular Washing Fluids}

Leaves of tobacco plants were immersed in $10 \mathrm{mM}$ sodium phosphate, $\mathrm{pH} 7.5$ and exposed to three periods of vacuum each lasting $5 \mathrm{~min}$, followed by release of the vacuum. Infiltrated leaves were gently dried by blotting with absorbed paper. Intercellular fluid was collected by centrifugation for $20 \mathrm{~min}$ at $1000 \mathrm{~g}$. The rest of the leaves were homogenized in plant extraction buffer to prepare the crude protein extract. Cell debris was removed by centrifugation $(16,000 \mathrm{~g} / 30 \mathrm{~min})$ and the supernatant was subjected to immunoblot analysis and determination of enzymatic activity. The primary 9E10 anti-c-myc antibody (1:5000) and the rabbit anti-Solanum- 
PEPC antibody (1:2000) were used to detect recombinant ODC and PEPC from IWF and crude extract fractions.

\section{Biochemical Characterization of Stably Transformed Tobacco}

Determination of ODC activity was performed as described $[25,26]$. Plant material used for the biochemical analysis was collected from leaves of same ontogenic stage (first well developed top leaf from 30-day-old plants), since the endogenous polyamine titers are significantly affected by the ontogenic stage of the leaves. The mean value of the ODC activity was expressed in $\mathrm{nmol} \mathrm{CO}_{2} / \mathrm{mg}$ total soluble protein/h. Analysis of free polyamine levels and quantification were performed as described by Nölke et al. [26]. Results were expressed as $\mathrm{nmol} / \mathrm{g}$ fresh weight $(\mathrm{FW})$.

\section{RESULTS}

\section{Transient Expression of Human ODC in Different Plant Cell Compartments}

The odc cDNA sequence was amplified from a human prostate cDNA library and introduced into two expression cassettes that directed the recombinant protein to the cytosol (pODC-cyt) and apoplast (pODC-apo), respectively (Fig. 1). The accumulation of recombinant ODC was verified by transient expression in tobacco leaves.

(A) pODC-cyt

\begin{tabular}{|c|c|c|c|c|}
\hline & ScoI & \multicolumn{2}{c}{ SalI } & \multicolumn{2}{c}{ XbaI HindIII } \\
\hline
\end{tabular}

(B) pODC-apo

\begin{tabular}{|c|c|c|c|c|c|}
\hline CHS & SP & odc & c-myc & His6 & Pw \\
\hline
\end{tabular}

Fig. (1). Plant expression cassettes for targeting ODC to the plant cell cytosol (pODC-cyt) (A) and apoplast (pODC-apo) (B). P35S: CaMV 35S promoter with double enhancer; $\Omega$ : omega leader region of TMV RNA; CHS: 5' untranslated region of chalcone synthase from Petroselinum hortense; SP: plant codon optimized signal peptide derived from the light chain of the TMV-specific murine antibody 24 [19]; Pw: 3' untranslated region of TMV RNA; c-myc: myc epitope; His6: his-6 tag.

Immunoblot analysis revealed similar recombinant protein levels and a distinct band of about $51 \mathrm{kDa}$ for the cytosolic and apoplastic ODC product, corresponding to the expected molecular weight of ODC (Fig. 2A). The identical size of the two, differentially-targeted proteins indicated correct cleavage of the apoplastic targeting signal. Furthermore, total RNA extracted from tobacco leaves transformed with the pODC-cyt or pODC-apo constructs was analysed by Northern blot to confirm the presence of corresponding transcripts. In both cases, a $1.5 \mathrm{~kb}$ transcript hybridized with the probe and generated a signal of approximately the same in- tensity, which was absent in RNA extracted from wild type tobacco (Fig. 2B).

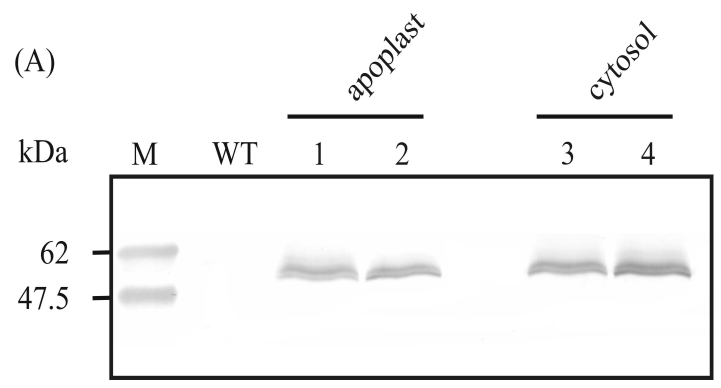

(B)

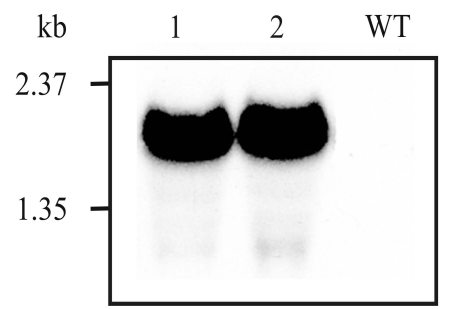

Fig. (2). Transient expression of recombinant ODC in tobacco leaves. (A): Immunoblot analysis of the crude total soluble protein (TSP) $(10 \mu \mathrm{g})$ extract of independent leaves transiently producing recombinant ODC targeted to the plant apoplast (lane 1 and 2) or cytosol (lane 3 and 4). The 9E10 anti-c-myc antibody was used for recombinant protein detection. M: prestained protein marker; WT: TSP extract from wild type tobacco leaves. (B): Northern blot analysis of transcript levels of apoplastic and cytosolic ODC. Total RNA was isolated from infiltrated leaves producing ODC in the apoplast (lane 1) or cytosol (lane 2) and from leaves of wild type tobacco plants (WT). Per lane $10 \mu \mathrm{g}$ total RNA was loaded and separated on a $1 \%(\mathrm{w} / \mathrm{v})$ agarose gel containing $2.4 \mathrm{M}$ formaldehyde prior to blotting onto a nylon membrane. DIG labelled $1.5 \mathrm{~kb}$ human odc gene was used as probe for hybridization. Detection of dioxigenin labelled ODC was performed by DIG luminescent detection kit for 2 min at RT.

\section{Transgenic Tobacco Plants Producing Human ODC}

To investigate effects of ectopic ODC expression in planta, the pODC-apo construct was introduced into tobacco plants by Agrobacterium-mediated transformation. Twentyfive $\mathrm{T}_{0}$ plants (named ODC-apo-1 to 25 ) were regenerated and screened for the presence of recombinant ODC. None of the transgenic plants had an unusual morphology and all set seed normally upon self-fertilization. To exclude metabolic variation due to somaclonal effects in plants derived from tissue culture $[27,28]$, transgenic $\mathrm{T}_{0}$ plants showing the highest accumulation of recombinant ODC (ODC-apo-7; 85 $\mu \mathrm{g}$ per $\mathrm{g}$ fresh weight) were propagated to the $\mathrm{T}_{1}$ generation. Accumulation of the recombinant enzymes in $T_{1}$ plants was comparable to that in parental lines. The $\mathrm{T}_{1}$ lines ODC-apo$7 / 3$ producing the highest level of recombinant protein (80$85 \mu \mathrm{g}$ per $\mathrm{g}$ fresh weight) were chosen for generation of homozygous lines. Homozygous $\mathrm{T}_{2}$ showed approximately two fold higher recombinant protein levels than the $T_{0}$ and $T_{1}$ generations (data not shown). 


\section{Localization of Recombinant ODC in the Apoplast of Tobacco Plants}

Previous studies have shown that the codon optimised Nterminal signal peptide used in this report facilitates the secretion of recombinant proteins into the apoplastic space [2931]. Therefore, we assumed that recombinant ODC was translocated into the endoplasmatic reticulum and passed through the secretory pathway eventually to be secreted to the apoplast.

However, to confirm the apoplastic localization of the recombinant enzyme, the intercellular washing fluid (IWF) was isolated from transgenic $\mathrm{T}_{1}$ line ODC-apo-7/3, one of the better-producing lines, and tested for the presence of recombinant ODC by immunoblot analysis and enzyme activity assays. To exclude the possibility that extraneous ODC could contaminate the IWF through cell damage the intercellular fluids of transgenic and wild type tobacco plants were also tested for the levels of the cytosolic marker phosphoenolpyruvate carboxylase (PEPC). Simultaneous detection of recombinant ODC and native PEPC by immunoblot confirmed the presence of recombinant ODC $(\sim 51 \mathrm{kDa})$ (Fig. 3). Importantly, PEPC was not detectable in the IWF of the transgenic and wild type lines (Fig. 3, lane 1 and 4), confirming that the recombinant ODC was not released from broken cells. In contrast, cytosolic PEPC (116 kDa) was detectable in total soluble proteins extracted from transgenic and wild type tobacco leaves subsequent to IWF isolation (Fig. 3, lanes 3 and 5). Analysis of enzymatic activity demonstrated that the ODC activity in the intercellular washing fluid was comparable to the ODC activity in the crude total soluble protein extract (data not shown). These results demonstrated that the N-terminal signal peptide we used was indeed efficient at translocating the recombinant ODC to the apoplast.

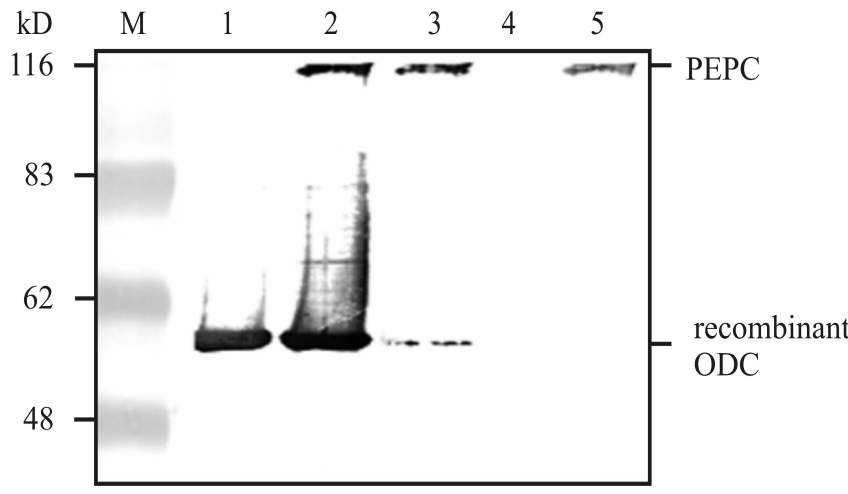

Fig. (3). Localisation analysis of recombinant human ODC in tobacco leaves. Immunoblot analysis of the intercellular washing fluid (IWF) and crude total soluble protein (TSP) of transgenic ODC-apo-7/3 and wild type tobacco leaves. Detection was performed with a combination of anti-c-myc (1:5000) and antiSolanum-PEPC (1:2000) antibodies, followed by addition of GAMAP (1:5000) and NBT/BCIP (nitroblue tetrazolium/5-bromo-4chloro-3-indolyl phosphate) substrate. $\mathrm{M}$ : prestained protein marker; $1: 15 \mu \mathrm{g}$ of protein of the intercellular washing fluid (IWF) collected from a transgenic ODC-apo-7/3 leaf; $2: 15 \mu \mathrm{g}$ of TSP extracted from a transgenic ODC-apo-7/3 leaf; 3: $15 \mu \mathrm{g}$ of TSP extracted from a transgenic ODC-apo-7/3 leaf subsequent to IWF collection; $4: 15 \mu \mathrm{g}$ of protein of the IWF collected from wild-type tobacco leaves; 5: $15 \mu \mathrm{g}$ of TSP extracted from a wild type tobacco leaf after IWF collection.

\section{Activity of Human ODC in Leaf Tissue}

To investigate whether the apoplastic human ODC is functional in planta, ODC activity was measured in leaves of 30-day-old plants from six selected $\mathrm{T}_{2}$ transgenic lines (ODC-apo7/3-3, -4, -7, -10, -11 and -15), which accumulate recombinant ODC to high levels (120-140 $\mu \mathrm{g}$ per $\mathrm{g}$ fresh weight) (Fig. 4A). Wild type tobacco plants were assayed under the same conditions. All transgenic plants showed a significant increase in ODC activity compared to wild type tobacco (Fig. 4B), and the increase in ODC activity correlated with rising ODC accumulation (correlation coefficient $\mathrm{R}^{2}=0.8356$; data not shown). The strongest increase in ODC activity was observed in line ODC-apo7/3-3 (32-fold; $\mathrm{P}<0.001)$ and ODC-apo7/3-11 (30-fold; $\mathrm{P}<0.001$ ), which also showed the highest levels (140 $\mu \mathrm{g}$ per $\mathrm{g}$ fresh weight) of ODC protein.

\section{Polyamine Levels in Plants Producing ODC-APO}

To examine the effect of apoplastic human ODC on polyamine metabolism, levels of free polyamines were measured in the vegetative tissue of $\mathrm{T}_{2}$ lines ODC-apo7/3-3, -4, -7, -10, -11 and -15 . Leaf material was collected simultaneously from all lines, to avoid different abiotic and biotic factors that may affect the polyamine metabolism and could confound the results. A significant increase in putrescine levels was observed in all transgenic lines compared to wild type tobacco plants (Fig. 4C).

The strongest increase was observed in lines ODCapo7/3-3 and ODC-apo7/3-11 ( $\mathrm{p}<0.05)$, which also showed the highest ODC activity. No significant variation $(\mathrm{P}>0.05)$ of spermidine and spermine levels was detected when compared to wild type plants (Fig. 4C). It is striking that the increase of putrescine levels strongly correlated with ODC accumulation and activity, indicating that expression of recombinant ODC was responsible for increased putrescine synthesis.

\section{DISCUSSIONS}

The polyamine biosynthetic pathway has been the subject of intensive studies for several decades. Strategies to manipulate the pathway are based on the overexpression and antibody- or RNA-based inhibition of key anabolic enzymes in concert with the down regulation of catabolic enzymes such as diamine oxidase (DAO) and polyamine oxidase (PAO) $[4,7,26,32,33]$. In several of these studies, only minor variations in polyamine levels were observed, suggesting that the levels of spermidine and spermine are under strict homeostatic regulation. Recently, we have shown that an antibody-based inhibition of ODC in tobacco plants exerts pleiotropic effects on the upstream and downstream steps in the pathway leading to a reduction in ODC activity in vivo, decreased levels of putrescine, spermidine and spermine and an aberrant morphological phenotype [26].

In this study, we investigated the effect of subcellular targeting on ODC accumulation in tobacco plants, in order to determine the potential of a targeting approach for the modulation of polyamine biosynthesis. Successful targeting of functional enzymes to appropriate subcellular compartments may provide an alternative method for the manipulation of the polyamine pathway. To our knowledge, this is the first 
report which has investigated the use of atypical subcellular targeting for this purpose.

(A)

(B)
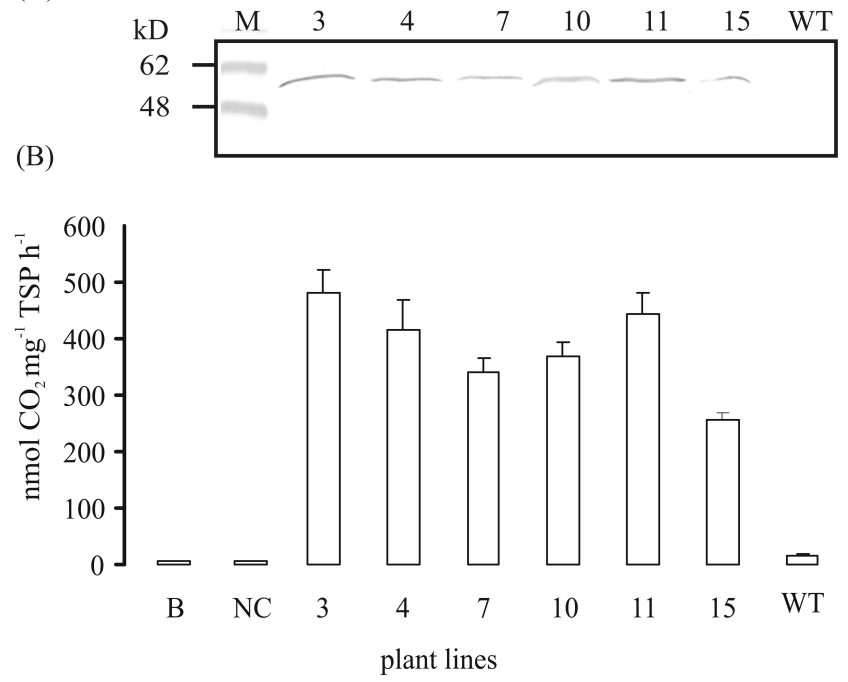

(C)

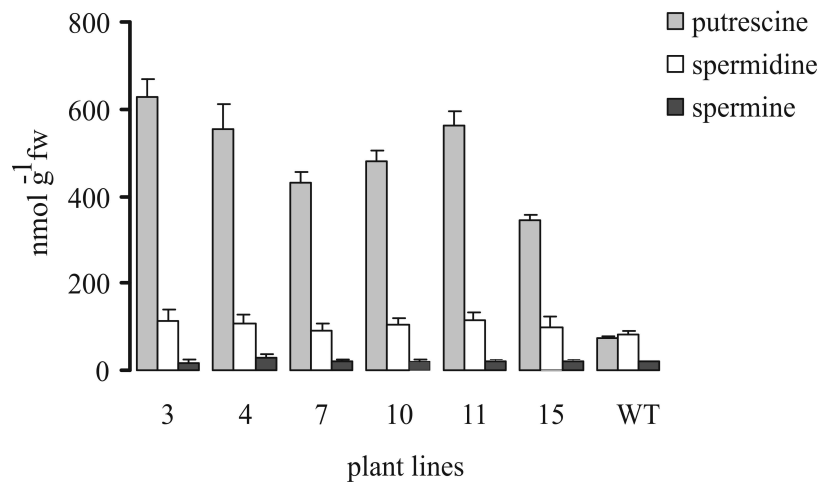

Fig. (4). Biochemical analysis of stably transformed tobacco plants accumulating human ODC in the plant cell apoplast. (A): Immunoblot analysis of total soluble protein (TSP) $(5 \mu \mathrm{g})$ extracted from six independent lines producing recombinant ODC. Plant lines $3,4,7,10,11,15$ : ODC-apo 7/3-3, -4, -7, -10, -11, -15 transgenic T2 lines; WT: wild type tobacco plant. (B): Enzymatic ODC activity analysis in wild type and transgenic $\mathrm{T} 2$ lines. Mean values $\pm \mathrm{SE}$ $(n=4)$ from five wild type plants (WT) and from six transgenic lines are shown. The mean value of the ODC activity was expressed in $\mathrm{nmol} \mathrm{CO} 2 / \mathrm{mg}$ total soluble protein/h. Determination of plant TSP was performed using Bradford assay. B, HEPES extraction buffer pH 7.0; NC: boiled transgenic plant extract used as negative control; WT: wild type tobacco plant; plant lines $3,4,7,10,11,15$ : ODC-apo7/3-3, -4, -7, -10, -11, -15 transgenic T2 lines. (C): Analysis of cellular polyamine levels in transgenic T2 lines. Mean values \pm SE $(n=3)$ from four wild type plants $(\mathrm{WT})$ and for six transgenic plants are presented. Plant lines 3, 4, 7, 10, 11, 15: ODC-apo7/3-3, $4,-7,-10,-11,-15$ transgenic T2 lines. WT: wild type tobacco plant.

The native compartment for ODC is the cytosol, although some ODC also accumulates in the nucleus [34-39]. ODC enzyme activity has also been detected in isolated mitochondria and chloroplasts [40]. The overexpression of recombinant ODC in the plant cell cytosol has been reported $[4,10$, 32]. Transgenic poplar (Populus nigra $x$ maximowiczzii) cells expressing a mouse odc gene showed elevated ODC activity and increased polyamine levels. Lepri et al. [13] reported the overexpression of a human odc cDNA in transgenic rice plants under the control of the Ubi-1 promoter, achieving a 1.4- to 4-fold increase in the levels of the three major polyamines in vegetative tissues and in seeds. So far there have been several reports on the overexpression of ODC in the cytosol of tobacco plants. Bastola et al. [11] expressed a mouse odc gene in carrot and transgenic tobacco under control of plant-specific promoters, which yielded in a 2.5-fold increase in cellular putrescine levels. Expression of yeast ODC in transgenic hairy root cultures of Nicotiana rustica led to a 1.5-to 2.5 fold increase in ODC activity and up to 2-fold increase in putrescine and nicotine content [41]. Expression of a C-terminally deleted mouse ODC cDNA in first-generation transgenic tobacco plants led to 2- to 3- fold increase in putrescine compared to untransformed control plants [10]. When the Datura stramonium ODC was expressed in transgenic tobacco plants, a 1.5-2.1-fold increase in putrescine content was observed in leaves and 1.1- to 1.3fold in flower buds. However, there were no changes in spermidine and spermine levels [6].

To investigate the biological effect of ectopic ODC targeting in planta, transgenic tobacco plants were generated in which the human ODC enzyme was targeted to apoplast. This atypical localization was confirmed by analysis of intercellular washing fluids by immunoblot (Fig. 3) and enzymatic activity (data not shown). Biochemical analysis of transgenic $\mathrm{T}_{2}$ plants producing recombinant apoplastic ODC demonstrated that the human enzyme was enzymatically active. The ODC activity in transgenic lines was 18- to 32fold higher compared to wild type plants, leading to a 4.68.4-fold $(\mathrm{P}<0.05)$ increase in putrescine levels. Molecular and biochemical analysis revealed a strong correlation between the level of recombinant ODC in the apoplast, the increased enzymatic activity and the elevated levels of putrescine, confirming that the ODC is functional in the apoplast and is responsible for the modulation of the polyamine metabolism. In contrast a linear correlation between ODC mRNA levels, enzymatic activity and polyamine accumulation was not observed when human ODC was directed to the cytosol of rice plants [13].

The activity of ODC relies on the presence of pyridoxal $5^{\prime}$-phosphate as a cofactor, and L-ornithine as a precursor, which are usually present in the cytosol, mitochondria and vacuole [42-44]. Although little is known about homeostatic regulation of ornithine pools in plants [12], recent data suggest the presence of a homeostatic mechanism that alters ornithine production concomitant with its increased use [45]. It is possible that the high-level accumulation of recombinant ODC in the apoplast sequesters L-ornithine to this compartment, leading to increased putrescine levels. Such a phenomenon has been demonstrated by investigators studying the immunomodulation of abscisic acid in plants [46, 47].

The apoplast is where the catabolic enzymes of the polyamines pathway - amine oxidases - are localised. Amine oxidases include the copper-containing amine oxidases $(\mathrm{CuAO}$; EC 1.4.3.6) oxidizing the diamines Put and cadaverine at the primary amino groups, and the flavin-containing polyamine oxidases, which oxidize Spd and Spm at their secondary amino groups [48]. Therefore, it is remarkable that the production of ODC in the apoplast resulted in up to 8.4-fold increase in putrescine levels when compared to wild type 
plants. In a previous study, Rea et al. [33] showed that despite the high levels and activity of the catabolic enzymes maize polyamine oxidise and pea copper amine oxidase, no changes in the levels of polyamines and lignins were observed in transgenic tobacco plants, suggesting either the tight regulation of polyamine levels or the differential compartmentalization of the two recombinant proteins and the bulk of endogenous polyamines [33]. In our study, it seems likely that the putrescine catabolic pathway was saturated due to the increased levels of putrescine generated by recombinant ODC.

In higher plants, cells are equipped with an efficient transport system for the uptake of the exogenous polyamines. Considering that polyamine transport plays an important role on the regulation of the cellular polyamines, we hypothesize that the putrescine produced in the apoplast is transported inside the cell through a putrescine-preferential transporter possibly initiated from the apoplastic putrescine accumulation and diffusion across a concentration gradient. This hypothesis is supported by a recent study in soybean demonstrating that not only the activity of polyamine biosynthestic enzymes, but also the translocation of the precursors can regulate the metabolism of polyamine [49]. The polyamine uptake at the cellular level is a very rapid active mechanism, reaching saturation after 1-2 min and the absorbed polyamines are mostly stored in vacuoles [50]. At least two polyamine transporters including the plasma membrane localized putrescine-preferential transporter (LmPOT1) have been identified in several eukaryotic cell types [51, 52].

Previous studies have shown that increased ODC activity leads to increased putrescine levels but is insufficient to increase spermidine and spermine levels $[6,10,11,41]$. Therefore, it has been suggested that homeostatic mechanisms efficiently accommodate increased ODC activity, and that control over polyamine biosynthesis occurs at multiple interdependent steps [6]. This hypothesis is supported by our data. The constitutive overexpression of apoplastic human ODC appears to be sufficient to elevate the putrescine pool, but tight control prevents the accumulation of spermidine and spermine. This probably reflects the excess of putrescine, which may induce the activity of diamine oxidase, an enzyme that removes the primary amino group from putrescine [48]. The question arises as to whether this tight regulation of spermidine and spermine biosynthesis is due to the pathway compensating for the increased putrescine level or due to the different subcellular localisations of SPDS, spermine synthase and S-adenosylmethionine decarboxylase. Therefore, the constitutive co-expression of recombinant ODC and SPDS in the same compartment, i.e. the apoplast, was attempted in a different study performed in our laboratory. The successful expression of both enzymes resulted in increased levels of putrescine (up to 5-fold) and spermidine (up to 4.7-fold), suggesting that metabolite flux can be shunted further downstream by increasing the levels of the subsequent enzymes in the pathway. The increase in putrescine and spermidine levels correlated with recombinant ODC and SPDS levels, indicating that the recombinant ODC and SPDS were functional in their non-native subcellular compartment and were responsible for the observed modulation of polyamine biosynthesis (data not shown).
In conclusion, it was shown that targeting recombinant ODC to the apoplast provides an alternative strategy to enhance enzyme accumulation, in vivo enzyme function and putrescine levels. Similar studies performed in our group involving the overexpression of tryptophan decarboxylase [16], provide further support to this strategy: i.e., that targeting enzymes to alternative compartments of the plant cell may significantly influence protein accumulation and enzymatic activity. This study shed further light on the complexity of polyamine biosynthesis in intact plants providing a basis for further manipulations of the pathway by differential subcellular targeting. This strategy may be particularly useful when applied to rate limiting enzymes in the production of desired metabolic products.

\section{ACKNOWLEDGEMENTS}

The authors gratefully acknowledge Dr. Flora Schuster for producing the transgenic tobacco plants, Prof. Dr. Paul Christou and Dr. Richard Twyman for critical reading of the manuscript.

\section{REFERENCES}

[1] Igarashi K, Kashiwagi K. Polyamines: mysterious modulation of cellular functions. Biochem Biophys Res Commun 2000; 271: 55964.

[2] Shantz LM, Pegg AE. Translational regulation of ornithine decarboxylase and other enzymes of the polyamine pathway. Int J Biochem Cell Biol 1999; 31: 107-22.

[3] Wallace HM, Fraser AV, Huges A. A prespective of polyamine metabolism. Biochem J 2003; 376: 1-14.

[4] Martin-Tanguy J. Metabolism and function of polyamines in plants: recent development (new approaches). Plant Cell Reg 2001; 34: 135-48.

[5] Capell T, Bassie L, Christou P. Progress in plant metabolic engineering. Curr Opin Biotechnol 2004; 15: 148-54.

[6] Mayer MJ, Michael AJ. Polyamine homeostasis in transgenic plants overexpressing ornithine decarboxylase induces ornithine limitation. J Biochem 2003; 134: 765-72.

[7] Franceschetti M, Fornale S, Tassonia A, Zuccherelli K, Mayer MJ, Bagni N. Effects of spermidine synthase overexpression on polyamine biosynthetic pathway in tobacco plants. J Plant Physiol 2004; 161: 989-1001.

[8] Burtin D, Michael AJ. Overexpression of arginine decarboxylase in transgenic plants. Biochem J 1997; 325: 331-7.

[9] Bhatnagar P, Minocha R, Minocha SC. Genetic manipulation of the metabolism of polyamines in poplar cells. The regulation of putrescine catabolism. Plant Physiol 2002; 128: 1455-69.

[10] DeScenzo RA, Minocha SC. Modulation of cellular polyamines in tobacco by transfer and expression of mouse ornithine decarboxylase cDNA. Plant Mol Biol 1993; 22: 113-27.

[11] Bastola DR, Minocha SC. Increased putrescine biosynthesis through transfer of mouse ornithine decarboxylase cDNA in carrot provides tolerance embryogenesis. Plant Physiol 1995; 109: 63-71.

[12] Bhatnagar P, Glasheen BM, Bains SK, et al. Transgenic manipulation of the metabolism of polyamines in poplar cells. Plant Physiol 2001; 125: 2139-53.

[13] Lepri O, Basie L, Safwat G, et al. Over-expression of a cDNA for human ornithine decarboxylase in transgenic rice plants alters the polyamine pool in a tissue-specific manner. Mol Genet Genomics 2001; 266: 303-12.

[14] Falco SC, Guida T, Locke M, et al. Transgenic canola and soybean seeds with increased lysine. Biotechnol 1995; 13: 557-82.

[15] Galili S, Guenoune D, Wininger S, et al. Enhanced levels of free and protein-bound threonine in transgenic alfalfa (Medicago sativa L.) expressing a bacterial feedback insensitive aspartate kinase gene. Transgenic Res 2000; 9: 137-44.

[16] Di Fiore S, Li Q, Leech MJ, et al. Targeting tryptophan decarboxylase to selected subcellular compartments of tobacco plants affects 
enzyme stability and in vivo function and leads to a lesion-mimic phenotype. Plant Physiol 2002; 129: 1160-9.

[17] Voss A, Niersbach M, Hain R, et al. Reduced virus infectivity in $N$. tabacum secreting a TMV-specific full-size antibody. Mol Breed 1995; 1: 15-26.

[18] Konz C, Schell J. The promoter of TL-DNA gene 5 controls the tissue-specific expression of chimaeric genes carried by a novel type of Agrobacterium binary vector. Mol Gen Genet 1986; 204: 382-96.

[19] Vaquero C, Sack M, Chandler J, et al. Transient expression of a tumor-specific single-chain fragment and a chimeric antibody in tobacco leaves. Proc Natl Acad Sci USA 1999; 96: 11128-33.

[20] Kapila J, De Rycke R, van Montagu M, Agenon G. An Agrobacterium mediated transient gene expression system for intact leaves. Plant Sci 1996; 122: 101-8.

[21] Horsch R, Fry JE, Hoffman N, Eicholtz D, Rogers S, Fraley R. A simple and general method for transferring genes into plants. Science $1995 ; 227: 1229-31$.

[22] Collinge DB, Milligan DE, Dow JM, Scofield G, Daniels MJ. Gene expression in Brassica campestris showing a hypersensitive response to the incompatible pathogen Xanthomonas compestris pv. vitians. Plant Mol Biol 1987; 8: 405-14.

[23] Chomczynsky P. One-hour downward alkaline capillary transfer for blotting of DNA and RNA. Anal. Biochem 1992; 201: 134-9.

[24] Bronstein I, Voyta JC, Vant Erve Y, Kricka LJ. Advances in ultrasensitive detection of proteins and nucleic acids with chemiluminescence: novel derivatized 1,2-dioxetane enzyme substrates. Clin Chem 1991; 37: 1526-7.

[25] Capell T, Escobar C, Lui H, Burtin D, Lepri O, Christou P. Overexpression of the oat arginine decarboxylase cDNA in transgenic rice (Oriza sativa L.) affects normal development patterns in vivo and results in putrescine accumulation in transgenic plants. Theor Appl Genet 1998; 97: 246-54

[26] Nölke G, Schneider B, Fischer R, Schillberg S. Immunomodulation of polyamine biosynthesis in tobacco plants has a significant impact on polyamine levels and generates a dwarf phenotype. Plant Biotechnol J 2005; 3: 237-47.

[27] Kaeppler SM, Kaeppler HF, Rhee Y. Epigenetic aspects of somaclonal variation in plants. Plant Mol Biol 2000; 43: 179-88.

[28] Courtial B, Feuerbach F, Eberhard S, et al. Tnt1 transposition events are induced by in vitro transformation of Arabidopsis thaliana, and transposed copies integrate into genes. Mol Genet Genomics 2001; 265: 32-42.

[29] Fischer R, Schumann D, Zimmermann S, Drossard J, Sack M, Schillberg S. Expression and characterization of bispecific singlechain Fv fragments produced in transgenic plants. Eur J Biochem 1999; 262: 810-16.

[30] Sriraman R, Bardor M, Sack M, et al. Recombinant anti-hCG antibodies retained in the endoplasmic reticulum of transformed plants lack core-xylose and core- $\alpha(1,3)$-fucose residues. Plant Biotechnol J 2004; 2: 279-87.

[31] Abranches R, Marcel S, Arcalis E, Altmann F, Fevereiro P, Stoger E. Plants as bioreactors: A comparative study suggest that Medicago truncatula is a promising production system. J Biotechnol 2005; 120: 121-34.

[32] Capell T, Bassie L, Christou P. Modulation of the polyamine biosynthesis pathway in transgenic rice confers tolerance to drought stress. Proc Natl Acad Sci USA 2004; 101: 9909-14.

[33] Rea G, de Pinto MC, Tavazza R, et al. Ectopic expression of maize polyamine oxidase and pea copper amine oxidase in the cell wall of tobacco plants. Plant Physiol 2004; 134: 1414-26.

[34] McCormick F. Polyamine metabolism in enucleated mouse L-cells. J Cell Physiol 1977; 93: 285-92.
[35] Panagiotidis CA, Georgatsos JG, Kyriakidis DA. Superinduction of cytosolic and chromatin-bound ornithine decarboxylase activities of germinating barley seeds by actinomycin D. FEBS Lett 1982 ; 146: $193-6$.

[36] Zagon IS, McLaughlin PJ, Seely JE, Hoeksema GW, Pegg AE. Autoradiographic localization of ornithine decarboxylase in mouse kidney by use of radiolabeled alpha-difluoromethylornithine. Cell Tissue Res 1984; 235: 371-7.

[37] Slocum DD. In: Slocum RD, Flores HE. Eds. Biochemistry and physiology of polyamines in plants: Tissue and subcellular localization of polyamines and enzymes of polyamine metanbolism. CRC Press, Boca Raton 1991; 93-105

[38] Tassoni A, Fornale N, Bagni N. Putative ornithine decarboxylase activity in Arabidopsis thaliana: inhibition and intracellular localization. Plant Physiol Biochem 2003; 41: 871-5.

[39] Schipper RG, Cuijpers VM, De Groot LH, Thio M, Verhofstad AA. Intracellular localization of ornithine decarboxylase and its regulatory protein, antizyme-1. Histochem Cytochem 2004; 52: 1259-266.

[40] Torrigiani P, Serafini-Fracassini D, Biondi S, Bagni N. Evidence for the subcellular localization of polyamines and their biosynthetic enzymes in plant cells. J Plant Physiol 1986; 124: 23-9.

[41] Hamill JD, Robins RJ, Parr AJ, Evan DM, Furze JM, Rhodes MJC Over-expression of a yeast ornithine decarboxylase gene in transgenic roots of Nicotiana rustica can lead to enhanced nicotine accumulation. Plant Mol Biol 1990; 15: 27-38.

[42] Shargool PD, Steeves T, Weaver M, Russell M. The localization within plant cells of enzymes involved in arginine biosynthesis. Can J Biochem 1978; 56: 273-9.

[43] Davis RH. Compartmental and regulatory mechanisms in the arginine pathways of Neurospora crassa and Saccharomyces cerevisiae. Microbiol Rev 1986; 50: 280-313.

[44] Green SM Eisenstein E, McPhie P, Hensley P. The purification and characterization of arginase from Saccharomyces cerevisiae. J Biol Chem 1990; 265: 1601-07.

[45] Paschalidis KA, Roubelakis-Angelakis KA. Spatial and temporal distribution of polyamine levels and polyamine anabolism in different organs/tissues of the tobacco plants. Correlations with age, cell division/expansion, and differentiation. Plant Physiol 2005; 138: $142-52$.

[46] Artsaenko O, Peisker M, Nieden U, et al. Expression of a single chain Fv antibody against abscisic acid creates a wilty phenotype in transgenic tobacco. Plant J 1995; 8: 745-50.

[47] Phillips J, Artsaenko O, Fiedler U, et al. Seed specific immunomodulation of abscisic acid activity induces a developmental switch. EMBO J 1997; 16: 4489-96.

[48] Federico R, Angelini R. In: Slocum RD, Flores HE, Eds. Biochemistry and Physiology of Polyamines in Plants: Polyamine catabolism. CRC Press, Boca Raton, 1991; 41-56.

[49] Ohe M, Kobayashi M, Niitsu M, Bagni N, Matsuzaki S. Analysis of polyamine metabolism in soybean seedlings using ${ }^{15} \mathrm{~N}$-labelled putrescine. Phytochemistry 2005; 66: 523-8.

[50] Bagni N, Pistocchi R. In: Slocum RD, Flores HE, Eds. Biochemistry and Physiology of Polyamines in Plants: Uptake and transport of polyamine metabolism in plants. CRC Press, Boca Raton, 1991; 105-18.

[51] Tomitori H, Kashiwagi K, Asakawa T, Kakinuma Y, Michael AJ, Igarahi K. Multiple polyamine transport systems on the vacuolar membrane in yeast. Biochem J 2001; 353: 681-8.

[52] Hasne MP, Ullman B. Identification and characterization of a polyamine permease from the protozoan parasite Leishmania major. J Biol Chem 2005; 280: 15188-94. 